\title{
A case of embryonal sarcoma of the liver
}

\author{
A Pathirana ${ }^{1}$, R C Siriwardana ${ }^{2}$, K I Deen ${ }^{3}$, Y Rupasinghe ${ }^{1}$ \\ (Index words: hepatic embryonal sarcoma, extended liver resection, liver hypertrophy)
}

\section{Case report}

A 32-year old male had a nine month history of worsening right hypochondrial pain, gradual abdominal distension, loss of weight and loss of appetite. On further evaluation his body mass index was 16.9. There was gross hepatomegaly. He was in Child's grade B (low albumin and ascites). Computerised tomography (CT) revealed a massive liver tumour involving segments IV, V, VI and VII (Figure 1). Compensatory hypertrophy was seen in segment II and III. Inferior venacava and the right branch of the portal vein were also compressed by the tumour.

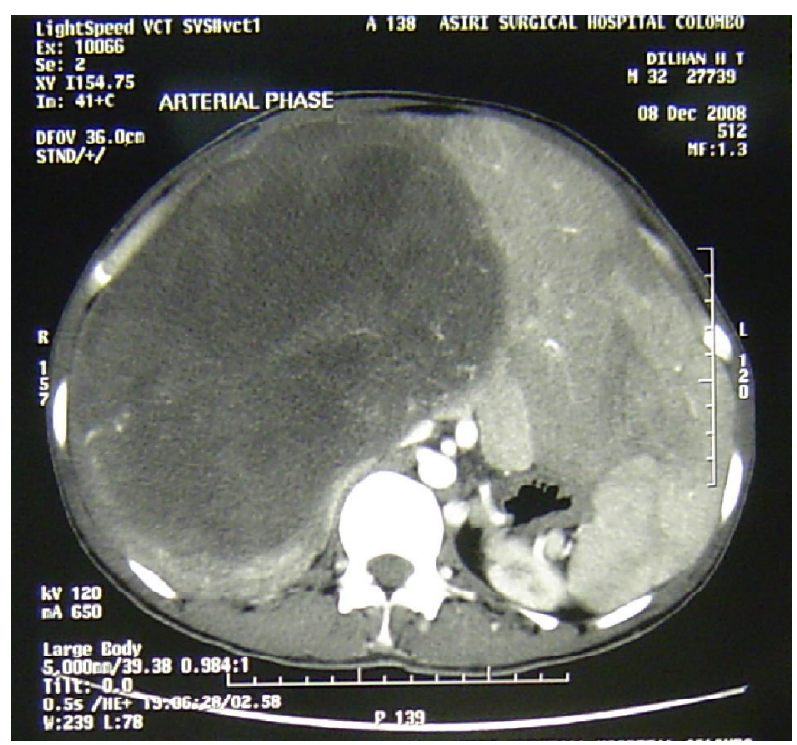

Figure 1. Tumour involving entire right lobe

An extended right hepatectomy was planned. A "Mercedes Benz" incision needed further extension into the right thorax for additional exposure. The Kelly clamp crushing technique was used for resection with intermittent cross clamping of inflow using Pringle's manoeuvre. A $4.7 \mathrm{~kg}$ mass was removed with total blood loss of 2.4l. The patient made an uneventful recovery. Histology revealed an embryonal sarcoma with microscopically clear resection margins.

\section{Discussion}

Adult embryonal sarcomas (ES) are rare. The first comprehensive analysis of ES included 31 cases [1]. Based on limited data available, surgical resection is known to offer the best chance of long-term cure. Four cases of complete resection of ES achieving more than 1 year survival has been reported [2]. The median survival is less than one year [1].

In extensive hepatic resections, at least $25 \%$ remaining liver volume is recommended to sustain normal hepatic function. If not, pre-operative portal vein embolisation is recommended. However, in this patient, occlusion of the right branch of the portal vein by the tumour, had led to hypertrophy of segment II and III. The largest tumour weight reported up to now is $3.4 \mathrm{~kg}$ in a post-mortem specimen [1]. Our case is the largest resected tumour reported.

Because ES is rare, no comprehensive data are available regarding the best form of therapy. Liver transplantation for ES is associated with a poor outcome due to recurrent disease in the transplanted liver [3] Chemotherapy has been used in both adjuvant and neoadjuvant settings [4].

\section{References}

1. Stocker JT, Ishak KG. Undifferentiated (embryonal) sarcoma of the liver. Cancer 1978; 42: 336-48.

2. Walker NI, Horn MJ, Strong RW, et al. Undifferentiated (embryonal) sarcoma of the liver. Cancer 1992; 69: 52-9.

3. Penn I. Hepatic transplantation for primary and metastatic cancers of the liver. Surgery 1991; 110: 726-34.

4. Kim DY, Kim KH, Jung SE, et al. Undifferentiated (embryonal) sarcoma of the liver: combination treatment by surgery and chemotherapy. Journal of Paediatric Surgery 2002; 37: 1419-23.

${ }^{1}$ Department of Surgery, University of Sri Jayawardanapura, Sri Lanka, ${ }^{2}$ Department of Surgery, North Colombo Teaching Hospital, Sri Lanka and ${ }^{3}$ Department of Surgery, University of Kelaniya, Sri Lanka.

Correspondence: RCS, e-mail <rohansiriwardana@yahoo.com>. Received on 14 October 2009 and revised version accepted on 5 March 2010. Competing interests: none declared. 